\title{
Flare induced penumbra formation in the sunspot of NOAA 10838
}

\author{
Sreejith Padinhatteeri ${ }^{1,2}$ and Sankarasubramanian K. ${ }^{1}$ \\ ${ }^{1}$ Space Astronomy Group, ISRO Satellite Centre, Bangalore, \\ India - 560017 \\ email: sreejith.p@gmail.com,sankark@isac.gov.in \\ ${ }^{2}$ Dept. of Physics, University of Calicut, Kerala, India.
}

\begin{abstract}
We have observed formation of penumbrae on a pore in the active region NOAA10838 using Dunn Solar Telescope at NSO,Sunpot,USA. Simultaneous observations using different instruments (DLSP,UBF,Gband and CaK) provide us with vector magnetic field at photosphere, intensity images and Doppler velocity at different heights from photosphere to chromosphere. Results from our analysis of this particular data-set suggests that penumbrae are formed as a result of relaxation of magnetic field due to a flare happening at the same time. Images in $\mathrm{H} \alpha$ show the flare (C 2.9 as per GOES) and vector magnetic fields show a re-orientation and reduction in the global $\alpha$ value (a measure of twist). We feel such relaxation of loop structures due to reconnections or flare could be one of the way by which field lines fall back to the photosphere to form penumbrae.
\end{abstract}

Keywords. Sunspot, penumbra, flare.

\section{Introduction}

Sunspots are the manifestation of strong magnetic fields that emerge in the solar photosphere (Bray \& Loughhead (1964), Solanki (2003)). Although sunspots are stable configurations when compared to the dynamical time scales of other features on the Sun, the observed umbral and penumbral fine-structures are very dynamic and subjected to constant change and transformation on small spatial scales. Our understanding of these processes and the nature of the fine structures improved significantly in the last decade (Rimmele (2008), Brummell et al. (2008)), but we still lack detailed knowledge about the key process of penumbral formation and decay. Observations of this process are very rare, most prominent among them are by Leka \& Skumanich (1998), Yang,et al. (2003) and Schlichenmaier et al. (2010). All of them observe the formation of a penumbra happening in few hours time. In all the cases they suggest the onset of penumbral formation is due the flux emergence. Leka \& Skumanich (1998) suggests critical flux limit of $1-1.5 \times 10^{20} \mathrm{Mx}$ for initiation of penumbra. Here in this paper we discuss multi-wavelength observations of the formation of penumbrae in the active region NOAA 10838 which was carried out on 2005 December 22. Coincidentally, a C-2.9 class flare was also observed in the same active region. These observations corresponding to different heights in the solar atmosphere suggest that the penumbral formation in this particular case is related to the flare at chromospheric and higher layers.

\section{Observations and Data Analysis}

The formation of the penumbra was observed on the leading sunspot of the active region NOAA 10838 on 2005 December 22 at the Dunn Solar Telescope in Sunspot,NM, USA. 

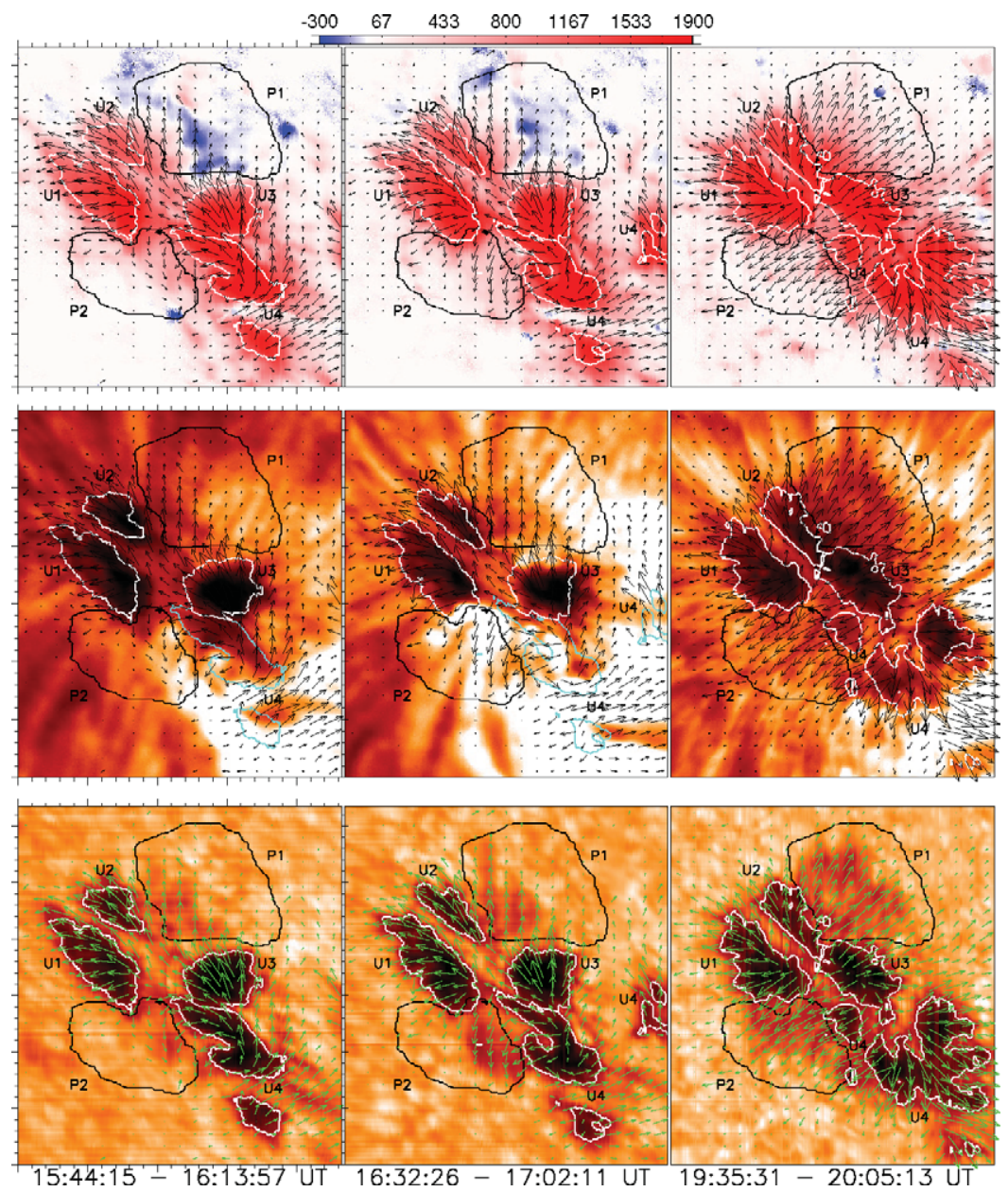

Figure 1. Figures showing Vector magnetic field evolution during the penumbral formation. Horizontal field vectors (arrows) are overplotted on vertical magnetic field (top row; with colour bar on the top showing the $B_{z}$ values), $\mathrm{H} \alpha$ image (middle row) and continuum image (bottom row). Raster scan time is mentioned at the bottom of each column. Dark contours mark the area where penumbra forms, and white contours mark different umbrae.

The observed sunspot was situated at $\mu=\cos \theta=0.77$ (N15.5 E35.4) on the solar disk. The atmospheric seeing was moderate during the observation. The adaptive optics system at the DST (Rimmele et al. (2004)) was operated and the atmospheric seeing corrected beam was fed to a set of back-end instruments. The main back-end instruments used were: Diffraction Limited Spectro-Polarimeter (DLSP; Sankarasubramanian et al. (2006)) and Universal Birefringent Filter (UBF; Beckers et al. (1975)). DLSP was used to obtain stokes profiles of two magnetically sensitive lines, Fe I $\lambda 6301.5 \AA$ and $6302.5 \AA$. The stokes profiles were inverted with an assumption of Milne-Eddington atmosphere using the HAO inversion code. The magnetic field vector components were transformed from observer's to heliographic co-ordinates using simple spherical trigonometric transformations (see Smart \& Green (1977)). The UBF is a tunable Lyot filter with a pass-band that varies between 120 to $250 \mathrm{~m} \AA$ in the visible wavelength of 5000 to $7000 \AA$. The UBF was tuned 


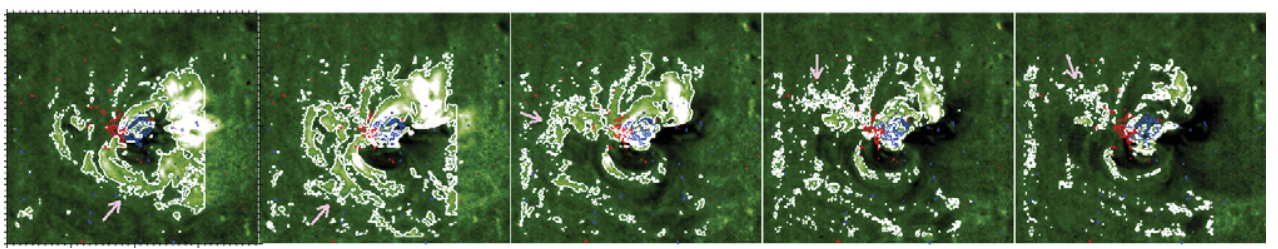

Figure 2. EIT images with white contours highlighting the bright loops. Red and blue contours represent positive and negative magnetic field, as derived from MDI, respectively. The arrow in each image show the loop that untwist as the flare happens.

in to two different spectral lines namely, Fe I $\lambda 5434$ and $\mathrm{H} \alpha \lambda 6562$. We have also used the archived data from EIT and MDI onboard SOHO satellite, during the same observation time, to study the loop dynamics during the flare. EIT and MDI data were calibrated and matched each other using standard codes available in the SSW software package. Magnetic field contours derived from MDI were overplotted on EIT images. To bring up the loop structure, a time averaged EIT image was subtracted from each frame and the intensity level contours were overplotted.

\section{Results and conclusion}

We have observed the formation of penumbrae, and a flare happening simultaneously. Preliminary results suggests a relation between the two. Top row of the Figure 1 shows the magnetic filed orientations at three raster scan maps. The colour code is used for vertical field strength and the arrows represent horizontal field. Dark Solid contours mark the ROI, selected manually, where penumbral formation happens, and are marked as $\mathbf{P} \mathbf{1}$ and P2. Four umbral areas are selected using the intensity values (less than $0.7 I_{Q S}$ where $I_{Q S}$ is the quite sun mean intensity), and are marked in white contours. U1, U2 and $\mathrm{U} 3$ are the bottom left, top left, and top right umbrae respectively. The bottom right umbra undergoes lot of changes, in shape and area, and many other small pores come and join. Hence all the umbral area other than U1, U2 and U3 are marked as U4. Apart from the bottom right umbra seen in first column, small pores which later join this spot are all grouped under U4. In the second row the horizontal field vector is overplotted on $\mathrm{H} \alpha$ line core images obtained using UBF at similar time of the respective raster scan. The flare can be seen as high intensity values in $\mathrm{H} \alpha$ images. In the third row horizontal field vector is overplotted on continuum raster scan image. The formation of penumbra is clearly visible in the continuum images. It is clear from the middle and bottom row images that the horizontal vectors match quite well with the magnetic structures like penumbra seen in continuum images and the super penumbral structures in $\mathrm{H} \alpha$ line core images. This suggest that the performed analyses, like inversions, azimuth ambiguity corrections and coordinate transformation from observers to heliographic co-ordinate were proper.

The arrows, representing the horizontal magnetic field, show a twisted field orientation in first two maps (first and second column of Figure 1), and as the flare happens, the field lines untwist to a more potential like nature as seen in third column. The global alpha value, $\boldsymbol{\alpha}_{\boldsymbol{g}}$, which is a measure of magnetic field twist was estimated (see for e.g. Tiwari et al. (2009)). The $\boldsymbol{\alpha}_{\boldsymbol{g}}$ of this sunspot reduces from $1.77 \times 10^{-7} / \mathrm{m}$ to $-0.43 \times 10^{-7} / \mathrm{m}$ as the penumbrae forms. The reduction in the $\boldsymbol{\alpha}_{\boldsymbol{g}}$ shows the relaxation of magnetic 
Table 1. Magnetic Flux $\Phi$ in units of $10^{20} \mathrm{Mx}$. Errors to Flux values in umbra are less than $0.05 \%$ and in penumbra are less than $0.12 \%$

\begin{tabular}{ccccccc}
\hline & U1 & U2 & U3 & U4 & P1 & P2 \\
\hline Map-1 & 1.67 & 0.50 & 1.45 & 2.44 & 0.37 & 0.89 \\
Map-2 & 1.96 & 0.61 & 1.55 & 2.65 & 0.45 & 0.99 \\
Map-3 & 2.06 & 1.02 & 1.89 & 3.76 & 1.58 & 0.74 \\
\hline
\end{tabular}

field configuration at the photosphere, due to the flare. The flare was of C-2.9 class as measured by GOES. Flare started at around 16:32 UT and peaked at around 16:50 UT.

The total longitudinal magnetic flux $\left(\sum f B_{z} A\right.$, where $f$ is the fill fraction, $A$ is the area and $B_{z}$ is the longitudinal magnetic field intensity) of this spot is of the order $10^{21}$ $\mathrm{Mx}$. It's little higher than the critical limit of $10^{20} \mathrm{Mx}$ needed for penumbral formation as suggested by Leka \& Skumanich (1998). There is an increase in the flux majorly due to similar polarity pores joining the main spot in U4. The flux values of four umbral region and the penumbral forming region are tabulated in Table 1. Apart from the flux values one also have to consider the twisted configuration of the magnetic fields. We believe that, in this case, the flare which transformed the twisted field structure to a more potential nature might have triggered the formation of penumbra.

Figure 2 gives the five images taken using EIT, with $\approx 12$ minutes cadence. The white contours shows the loops, and the blue and red contours represent the negative and positive magnetic field strength, derived from MDI, respectively. The images were taken close to the peak of the flare. The images show untwisting of large loops, in the same direction as seen in the photosphere vector magnetic field configuration. Similar untwisting is also seen in $\mathrm{H} \alpha$ time sequence movie. It suggest that the untwisting of magnetic field and the subsequent relaxation happens all the way from corona to photosphere.

We conclude that, observation of this particular event on this sunspot suggest a relation between flare and penumbral formation. Apart from the coincidence of both events, the observation also confirms the magnetic field re-orientation, all the way from corona to photosphere. Hence, we speculate that the penumbral formation may not be simply a photospheric phenomena but a result of global reconfiguration of magnetic field due to activities like a flare.

Acknowledgements: $\mathrm{SOHO}$ is a project of international cooperation between NASA and ESA. The NSO is operated by the AURA under a cooperative agreement with NSF.

\section{References}

Beckers, J. M., Dickson, L., \& Joyce, R. S., 1975, AFCRL Report No. AFCRL-TR-75-0090, A ir Force Cambridge Research Laboratory, Massachusetts.

Bray, R. J. \& Loughhead, R. E. 1964, Sunspots (The International Astrophysics Series, London: Chapman Hall, 1964)

Brummell, N. H., Tobias, S. M., Thomas, J. H., \& Weiss, N. O. 2008, ApJ, 686, 1454

Leka, K. D. \& Skumanich, A. 1998, ApJ, 507, 454

Rimmele, T. 2008, ApJ, 672, 684

Rimmele, T. R., Richards, K., Hegwer, S., et al. 2004, in SPIE Conf. Ser., eds. S. Fineschi \& M. A. Gummin, SPIE Conf. Ser., 5171, 179

Sankarasubramanian, K., Lites, B., Gullixson, C., et al. 2006, in ASP Conf. Ser., eds. R. Casini \& B. W. Lites, ASP Conf. Ser., 358, 201

Schlichenmaier, R.,Rezaei, R., González, N. B., \& Waldmann, T. A. 2010, A\&4A, 512, L1 
Smart, W. M. \& Green, R. M. 1977, Textbook on Spherical Astronomy, (Cambidge University Press, Cambidge, 1977)

Solanki, S. K. 2003, A\&AR, 11, 153

Tiwari, S. K., Venkatakrishnan, P., Gosain, S., \& Joshi, J. 2009, ApJ, 700, 199

Yang, G., Xu, Y., Wang, H., \& Denker, C. 2003, ApJ, 597,1190 